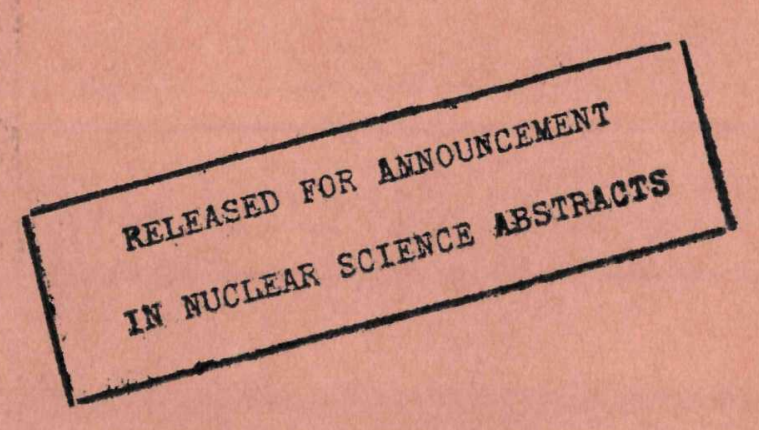

$$
\text { WAPD-TM-645 }
$$

AEC RESEARCH AND DEVELOPMENT REPORT

\title{
DIFFERENCE EQUATIONS FOR THE \\ NEUTRON DIFFUSION EQUATIONS \\ IN HEXAGONAL GEOMETRY
}

OCTOBER 1966

CONTRACT AT-11-1-GEN-14

BETTIS ATOMIC POWER LABORATORY, PITTSBURGH, PA., OPERATED FOR THE U. S. ATOMIC ENERGY COMMISSION BY WESTINGHOUSE ELECTRIC CORPORATION 


\section{DISCLAIMER}

This report was prepared as an account of work sponsored by an agency of the United States Government. Neither the United States Government nor any agency Thereof, nor any of their employees, makes any warranty, express or implied, or assumes any legal liability or responsibility for the accuracy, completeness, or usefulness of any information, apparatus, product, or process disclosed, or represents that its use would not infringe privately owned rights. Reference herein to any specific commercial product, process, or service by trade name, trademark, manufacturer, or otherwise does not necessarily constitute or imply its endorsement, recommendation, or favoring by the United States Government or any agency thereof. The views and opinions of authors expressed herein do not necessarily state or reflect those of the United States Government or any agency thereof. 


\section{DISCLAIMER}

Portions of this document may be illegible in electronic image products. Images are produced from the best available original document. 
WAPD-TM-645

UC-32: Mathematics and Computers

Special Distribution

CRBI PDICES

\section{DIFFERENCE EQUATIONS FOR THE NEUTRON DIFFUSION}

EQUATIONS IN HEXAGONAL GEOMETRY

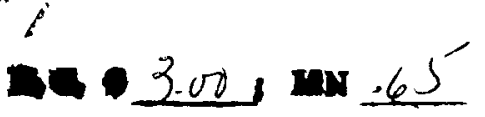

R. B. Kellogg

October 1966

REIEASED FOR ANNOUNCEXENT

IN NUCIEAR SCIENCE ABSTRACTS

CONTRACT AT-11-1-GEN-14

For sale by the Clearinghouse for Federal Scientific and Technical Information, National Bureau of Standards, U.S. Department of Commerce, Springfield, Va.

This document is an interim memorandum prepared primarily for internal reference and does not represent a final expression of the opinion of Westinghouse When this memorandum is distributed externally, it is with the express understanding that Westinghouse makes no representation as to completeness, accuracy, or usability of information contained therein. 
AEC Washington, J. M. Simmons

Argonne National Iaboratory, W. F. Miller

Atomics International

Babcock and Wilcox Company

Battelle Memorial Institute

Battelle-Northwest

Brookhaven National Iaboratory

Combustion Engineering, Inc. (IRD)

David Taylor Model Basin, H. Polachek

Du Pont Company, Aiken

General Atomic Division

General Electric Company, Cincinnati

General Electric Company, San Jose

Harvard University, G. Birkhoff

IIT Research Institute

Iowa State University

Knolls Atomic Power Laboratory, R. Ehrlich

Los Alamos Scientific Laboratory, B. Carlson

Mound Laboratory

National Reactor Testing Station (PPCO)

Naval Research Laboratory

New York University, R. Richtmyer

Nuclear Materials and Equipment Corporation

Union Carbide Corporation (ORNL), A. S. Householder

Union Carbide Nuclear Company (ORGDP), V. E. Anderson

University of Calffornia, Livermore, S. Fernbach

University of Maryland, R. B. Kellogg

Westinghouse Electric Corporation

DTIE

Manager, PNR
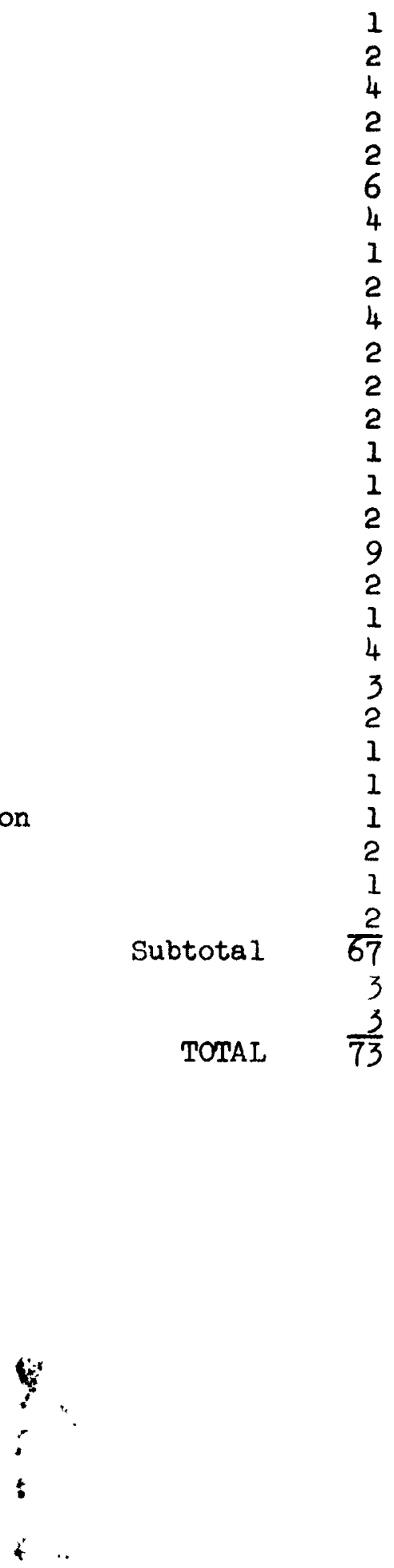
This report was prepared as an account of Government sponsored work. Neither the United States, nor the Commission, nor any person acting on behalf of the Commission:

A. Makes any warranty or representation, expressed or implied, with respect to the accuracy, completeness, or usefulness of the information contained in this report, or that the use of any information, apparatus, method, or process disclosed in this report may not infringe privately owned rights; or

B. Assumes any liabilities with respect to the use of, or for damages resulting from the use of any information, apparatus, method, or process disclosed in this report.

As used in the above, "person acting on behalf of the Commission" includes any employe or contractor of the Commission, or employe of such contractor, to the extent that such employe or contractor of the Comission, or employe of such contractor, prepares, disseminates, or provides access to, any information pursuant to his employment or contract with the Commission, or his employment with such contractor. 
The difference equations used in the hexogonal geometry option of the PDQ-7 program are derived. These difference equations are interpreted as belonging to a whole family of hexagonal difference approximations which in turn are derived using discontinuous trial functions. The truncation error of the PDQ-7 hexagonal difference equations is also discussed.

\section{DIFFERENCE EQUATIONS FOR THE NEUTRON DIFFUSION EQUATIONS IN HEXAGONAL GEOMEIRY}

R. B. Kellogg

\section{Introduction}

The purpose of this report is to derive the difference equations used in the hexagonal geometry option of PDQ-7. These equations, which were proposed by R. C. Gast, are interpreted as belonging to a whole family of hexagonal difference appoximations (Section II) which in turn are derived using discontinuous trial functions (Section IV) and for which an analysis of discretization error is presented (Section VI)。 In Section III the coefficients of the difference equations are displayed in a form which it is hoped is more suitable for programming. In section $V$ the truncation error of the difference equations is studied.

The PDQ-7 difference quations have also been used in magnetic field calculations [l]. The general imily of bexagonal difference approximations also includes the difference approximation of MacNeal $[2,3]$ which was used in PDQ-5T, and which seems to have been the first difference approximation proposed for use on a mesh arising from a general triangulation.

The leakage approximations in all these difference equations are the same; the only difference is in the treatment of the absorption term. The 
Ritz aifference approximation used in $P D Q-6[4,5,6]$ is aifferent from the approximations considered here in that the absorption term in the Ritz approximation does not give rise to a diagonal matrix.

The accuracy of the MacNeal approximation was discussed in [3], the accuracy of the Ritz approximation was discussed in [7], and the accuracy of the general class of difference approximations considered here is discussed in sections V and VI. All of the results obtained, however, give essentially the same error estimate. We know of no analysis which points to one of the schemes as being better than, or worse than, any of the others. 
II. A Class of Difference Approximations

We will consider difference approximations to the one group neutron diffusion equation in $(x, y)$ geometry

$$
-\underline{\nabla} \cdot(\underline{\nabla} \phi)+\sigma \phi=S,(x, y) \in R,
$$

$$
\phi \frac{\partial \phi}{\partial n}=0,(x, y) \in \partial R
$$

where $R$ is a domain in the $(x, y)$ plane, and where $\partial \phi / \partial n$ means the directional derivative of $\phi$ in the outward pointing normal direction of $\partial R$. The usual continuity of flux and current across region interfaces is specified as part of the boundary value problem

$$
\begin{aligned}
\phi^{+} & =\phi^{-} \\
D^{+}\left(\frac{\partial \phi}{\partial n}\right)^{+} & =D^{-}\left(\frac{\partial \phi}{\partial n}\right)^{-}
\end{aligned}
$$

where the t signs refer to the two sides of the region interface and $\underline{n}$ is a unit normal rector to the region interface.

The hexagonal mesh used in PDQ-7 is described in $[4,8]$. Basically, one starts with parallelogram (see Fig.1). Mesh lines are drawn parallel to the two sides of the parallelogram, forming a set of smaller parallelograms. The parallelograms are further divided into triangles. In this maner a triangulation of the original parallelogram is obtained. Parallelograms are now joined and overlaid to form a triangulation of the region $R$ in which the problem $(2.1),(2.2)$ is to be solved. 


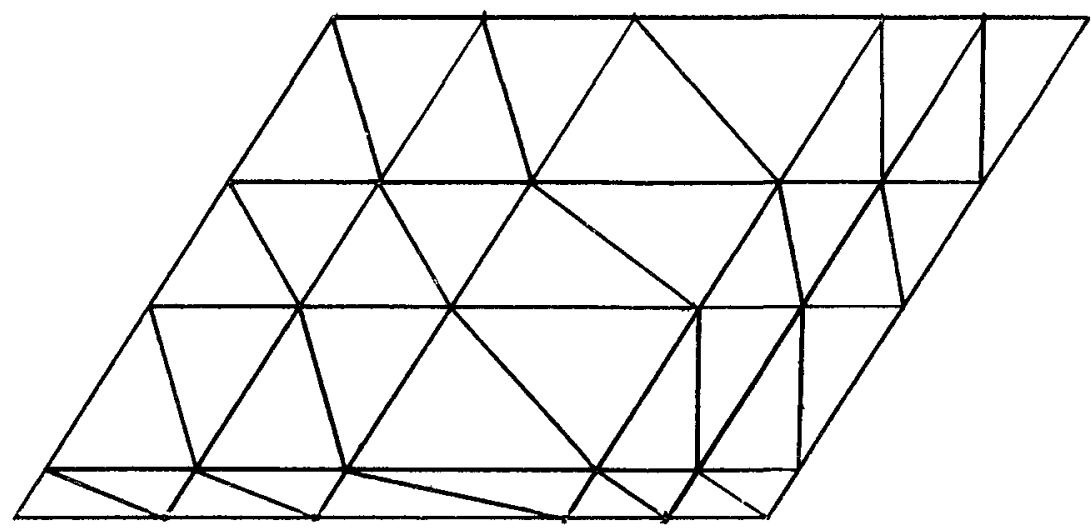

Figure 1

For the purpose of describing the dfference approximations, it is not necessary to use the detailed features of the mesh layout. We merely assume that the boundary $\partial R$ os $R$ is a polygon, and that $R$ is triangulated, decomposed into triangles, such that any two triangles hsve either no points in common, a vertex in common, or a side in common. We also assume that the coefficients $D$ and $\sigma$ are constant in each triangle of the triangulation. $T$ will denote the collection of triangles, $T$, in tide triangulation, and $\mathcal{f}$ will denote the collection of vertices of triangles $i$ in $J$. By construction, the vertices of the polygon $\partial \mathrm{R}$ must be included in the set $\mathcal{f}$. Two points, $P$ and $Q$, in $\mathcal{f}$ will be called neighbors of one another if there is a single triangle $\mathbb{T} \in \mathcal{J}$ such that both $\mathrm{F}$ and $Q$ are vertices of $T$. The set $\mathcal{f}$ is the set of mesh points of the problem.

It always happens in PDQ-7 that a mesh point $P$ has axactly 6 nefghbors unless $P$ lies on the boundary $\partial R$. A mesh point $P$ and 1ts 6 neighbors are depicted in Fig.2. To describe the general class of difference approximations considered in this report we suppose thst there is given for each 
triangle $T \in \mathcal{J}$ a point $B_{T} \in T$. The difference approximation depends upon the selection of points $B_{T}$. In Fig. 2 these are the points $B_{i}$. The points

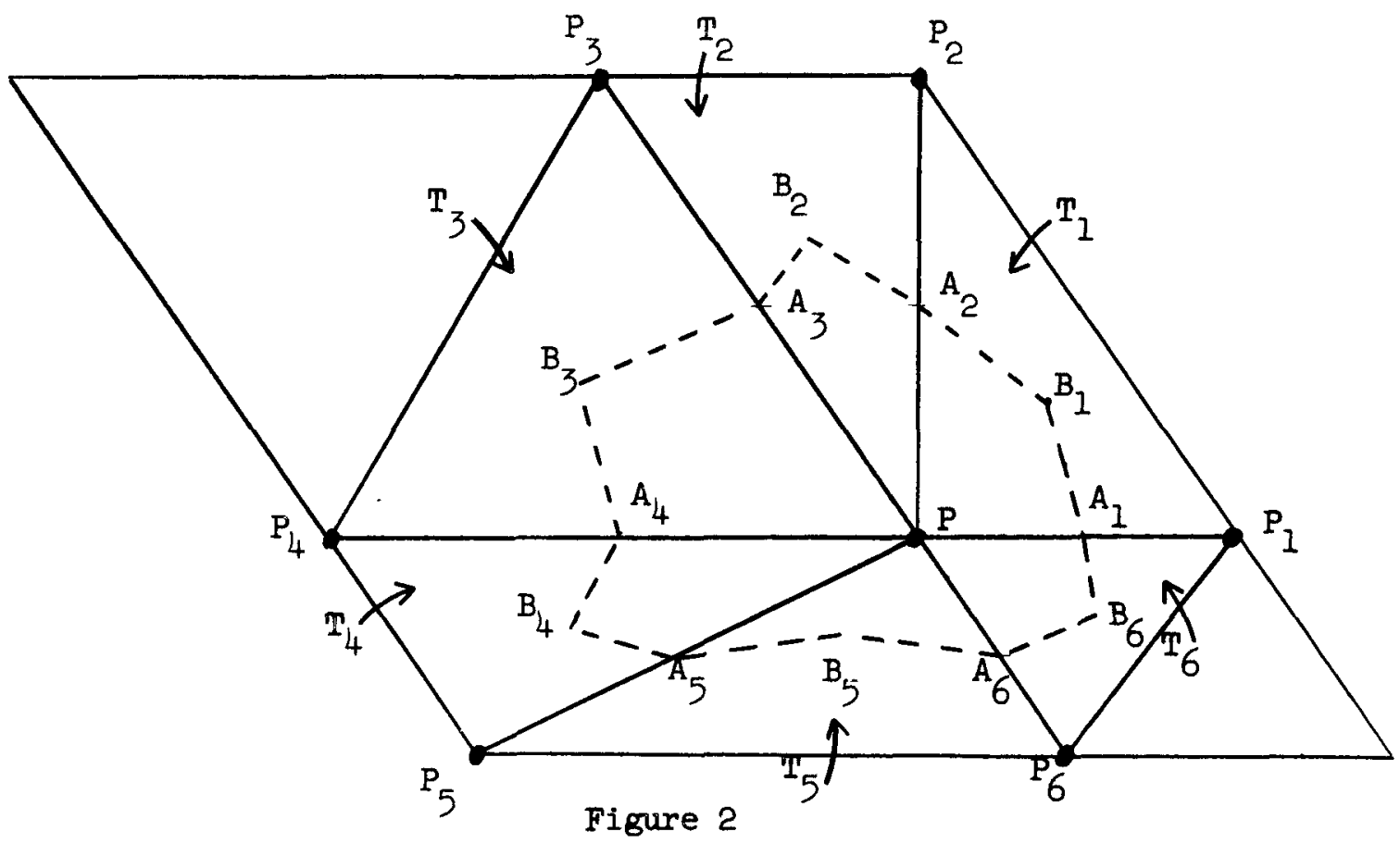

$A_{i}$ bisect the lines $\mathrm{PP}_{1}$, and the dodecagon joining the points $A_{1}, B_{1}$ is shown by the dotted line. This dodecagon, denoted by $\hat{a}(P)$, is the union of the 6 quadrilaterals

$$
a_{T_{i}}(P)=\hat{a}(P) \cap T_{i}, I \leq i \leq 6 .
$$

The areas of the dodecagon and the quadrilaterals are denoted respectively by $\hat{a}(P) i$ and $\left|a_{\mathbb{T}_{1}}(P)\right|$.

If both sides of $(2.1)$ are integrated over each quadrilateral $a_{T_{1}}(P)$, the leakage term is transformed into a line integral, and if the result is summed over $1=1, \ldots, 6$, the line integrals along $\mathrm{PA}_{1}$ cancel due to the continuity of current and there is obtained the equation 
$(2.3)$

$$
\sum_{i=1}^{6}\left\{-D_{1} \int_{A_{1}}^{B_{i}} \underline{N}_{i}^{\prime} \cdot \nabla \phi d s-D_{1} \int_{B_{1}}^{A_{1+1}} \underline{N}_{-1}^{v} \cdot \nabla \phi d s+\sigma_{1} \iint_{a_{T_{1}}} \phi d x d y\right\}
$$

$$
=\iint_{\hat{\mathbf{a}}(P)} \operatorname{sdxdy}
$$

where $D_{1}$ and $\sigma_{1}$ are the values of $D$ and $\sigma$ in $\mathbb{P}_{1}$, and where $N_{-1}^{\prime}$ and $N_{-1}^{n}$ are the unit normal vectors shown in Fig. 3.

In a typical mesh triangle $\mathbb{T}_{1}$, which is eituated with respect to the coordinate axes as in Fig. 3, the flux $\phi(x, y)$ mely be approximated by the linear expression

$$
\left\{\begin{aligned}
\phi(x, y) & \approx \hat{\phi}_{\mathrm{T}_{1}}(\mathrm{x}, \mathrm{y}), \\
\hat{\phi}_{\mathrm{T}_{1}}(\mathrm{x}, \mathrm{y}) & =\phi_{0}\left\{1-\frac{\mathrm{x}}{\mathrm{d}_{1}}-\frac{\mathrm{d}_{1}-\mathrm{d}_{1+1} \cos \alpha_{1}}{\left.\mathrm{~d}_{1} \mathrm{~d}_{1+1}{ }^{\sin \alpha_{1}} \mathrm{y}\right\}}\right. \\
& +\phi_{1}\left\{\frac{\mathrm{x}}{\mathrm{d}_{1}}=\frac{\mathrm{y}}{\mathrm{d}_{1} \operatorname{tgn\alpha _{1}}}\right\}+\frac{\mathrm{y}}{\mathrm{d}_{1+1} \sin \alpha_{1}} \phi_{1+1},
\end{aligned}\right.
$$

where we have used the notation

$$
\phi_{0}=\phi(P), \phi_{i}=\phi\left(P_{i}\right), \phi_{1+1}=\phi\left(P_{i+1}\right) .
$$

This suggests the approximation

$$
\nabla \phi \approx \nabla \hat{\phi}_{\mathrm{T}_{i}}=\frac{\phi_{1}-\phi_{0}}{a_{i}} \underline{1}+\left\{\frac{\phi_{i+1}-\phi_{0}}{a_{1+1} \sin \alpha_{i}}-\frac{\phi_{1}-\phi_{0}}{a_{i} \tan \alpha_{i}}\right\} \underline{j}
$$

for the gradient in mesh triangle $m_{1}$ 。 


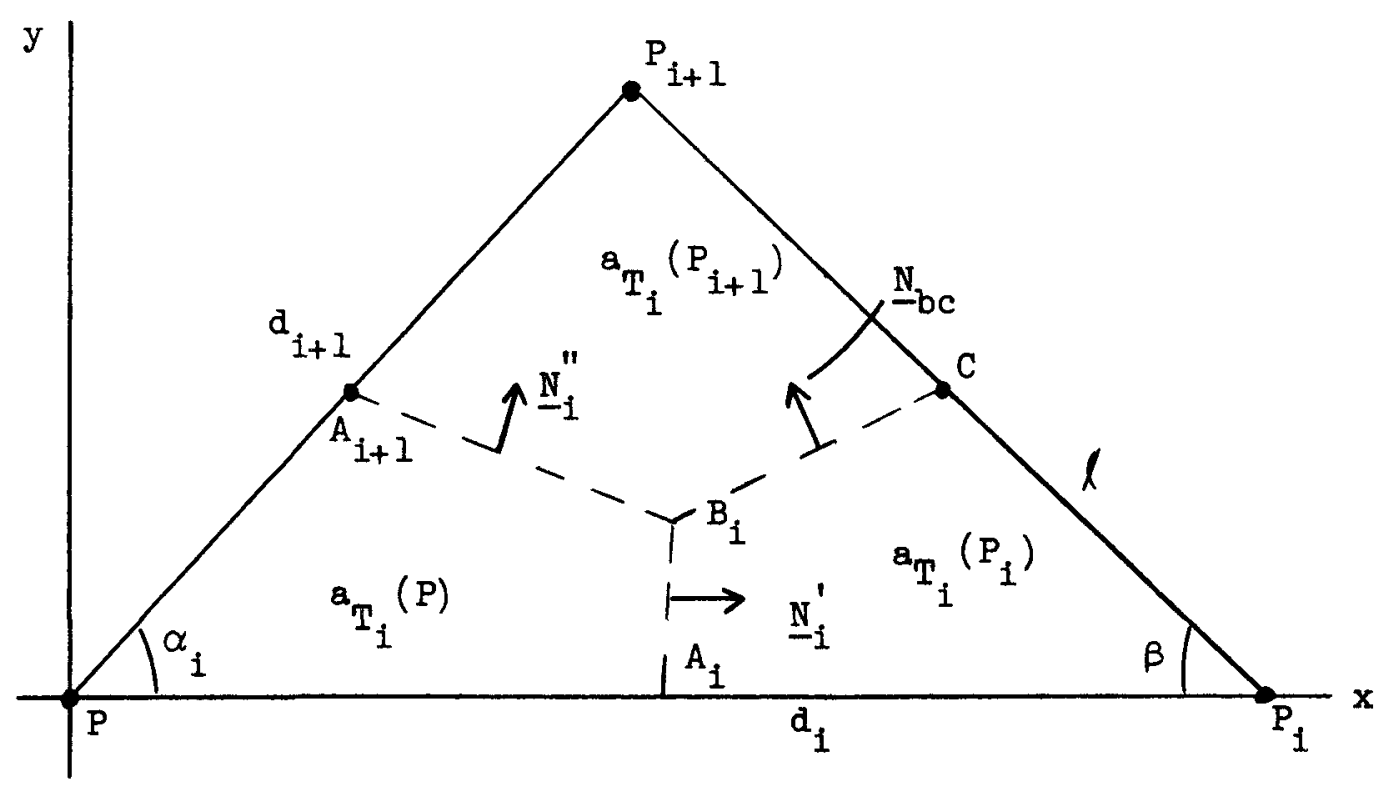

Figure 3

The approximation (2.5) is used in the leakage terms of (2.3). The absorption terms are approximated by assuming that $\phi$ is constant in $a_{T_{1}}(P)$. The resultant difference equation may be written

$$
\begin{aligned}
& \sum_{i=1}^{6} \int_{i} \frac{d_{i+1}-d_{1} \cos \alpha_{1}}{2 d_{i} \sin \alpha_{i}}\left(\tilde{\phi}_{0}-\tilde{\phi}_{i}\right) \\
& \left.\quad+D_{1} \frac{d_{i}-d_{i+1} \cos \alpha_{i}}{2 d_{i+1} \sin \alpha_{1}}\left(\tilde{\phi}_{0}-\tilde{\phi}_{i+1}\right)+\sigma_{1}\left|a_{T_{1}}(P)\right| \tilde{\phi}_{0}\right\} \\
& \quad=\iint_{\hat{\theta}(P)} \operatorname{sdxdy,}
\end{aligned}
$$

where $\tilde{\phi}_{0}, \tilde{\phi}_{1}, \tilde{\phi}_{i+1}$ are the approximate values of the flux at the mesh points $P, P_{1}$, and $P_{1+1}$ respectively. It may be noted that in (2.6) the leakage terms (the terms containing $D_{1}$ as a factor) do not depend upon the location of the point $B_{1}$. 
At a boundary point $P$ where $\phi_{0}$ is not known, the difference equation is obtained in a similar way. In this case, (2.3) and (2.6) are modified by summing only over those $i$ such that $a_{T_{i}}(P) \subset \bar{R}$. The integral of the source is now taken over $\hat{a}(P) \cap R$. In this manner, an equation (2.6) is obtained for each unknown flux $\phi(P)$. These equations may be represented collectively by the matrix equation

$$
\tilde{A Q}=\underline{s}_{1}
$$

where $\tilde{\emptyset}$ is the vector of approximate flux values. The matrix $A$ is symmetric and positive definite. The symmetry follows from the trigonometric identity (referring to Fig. 3)

$$
\frac{d_{1+1}-d_{1} \cos \alpha_{1}}{2 d_{1} \sin \alpha_{1}}=\frac{l-d_{1} \cos \beta}{2 d_{1} \sin \beta} .
$$

We now show that $\mathrm{A}$ is positive definite. Let $\mathscr{f}_{0}$ denote those mesh points $P$ of the triangulation such that $P \notin \partial R$ or such that, if $P \in \partial R$, then the boundary condition at $P\left(\right.$ see $(2.2)$ ) is $\partial \phi / \partial n=0$. (That is, $f_{0}$ is the collection of mesh points at which the value of the flux $\phi$ is not known.) Let $C_{0}$ be the collection of mesh functions $\Psi$ defined on $\&$ such

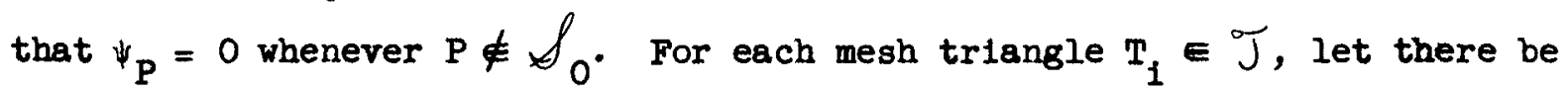
defined a nonnegative quadratic form $I_{T_{i}}(\Psi)$ on vectors $\Psi \in C_{0}$ by the formula

$$
\begin{aligned}
I_{T_{1}}(\Psi) & =D_{1}|T|\left\{\frac{1}{d_{1}^{2}}\left(\psi_{1}-\psi_{0}\right)^{2}+\left[\frac{\psi_{i+1}-\psi_{0}}{d_{i+1} \sin \alpha_{1}}-\frac{\psi_{i}-\psi_{0}}{a_{1} \tan \alpha_{i}}\right]^{2}\right\} \\
& +\sigma_{1}\left\{\left|a_{T_{1}}(P)\right| \psi_{0}^{2}+\left|a_{T_{1}}\left(P_{1}\right)\right| \psi_{1}^{2}+\left|a_{T_{1}}\left(P_{1+1}\right)\right| \psi_{i+1}^{2}\right\} ;
\end{aligned}
$$


the mesh triangle $T_{i}$ is as depicted in $F i g \cdot 3$, and $T_{i}=\frac{1}{2} d_{1} d_{1+1}$ sind $\alpha_{i}$ is the area of $T_{i}$. Note that $I_{T_{i}}(\Psi)$ is independent of which angle in Fig. 3 is called the angle $\alpha_{1}$. For example, we may also write

$$
\begin{aligned}
I_{T_{1}}(\Psi) & =D_{1}\left|T_{1}\right|\left\{\frac{1}{l^{2}}\left(\psi_{i+1}-\psi_{1}\right)^{2}+\left[\frac{\psi_{0}-\psi_{1}}{d_{1} \sin \beta}-\frac{\psi_{i+1}-\psi_{i}}{l \tan \beta}\right]^{2}\right\} \\
& +\sigma_{1}\left\{\left|a_{T_{1}}(P)\right| \psi_{0}^{2}+\left|a_{T_{1}}\left(P_{1}\right)\right| \psi_{1}^{2}+\left|a_{T_{1}}\left(P_{1+1}\right)\right| \psi_{1+1}^{2}\right\}
\end{aligned}
$$

Lemma 1. If $\Psi \subset C_{0}$, then

$$
\Psi^{T} A \Psi=\sum_{T \in \mathcal{T}} I_{T}(\Psi),
$$

and hence $A$ is positive definite.

$$
\begin{aligned}
& \text { Proof. Writing } \Psi^{T_{A \Psi}}=\sum_{R, Q \in \mathcal{S}_{0}}{ }^{a_{R Q} \psi_{R}{ }^{\psi}} \text {, we have } \\
& \frac{\partial}{\partial \psi_{P}} \underline{\Psi}^{\mathrm{T}} \underline{\underline{\Psi}}=2 \sum \mathrm{a}_{P Q} \psi_{Q}=2\left(\mathrm{~A}_{\underline{P}}\right)_{P}
\end{aligned}
$$

We will verify that the right side of (2.10) has the same derivative with respect to $\Psi_{P}$. Let $\sigma_{P}$ denote the set of all mesh triangles $T$ such that $P$ is a vertex of $T$. Then, referring to Fig. 2,

$$
\begin{aligned}
& \frac{\partial}{\partial \psi_{P}} \sum_{T \in \mathcal{T}} I_{T}(\Psi)=\sum_{T \in \sigma_{P}} \frac{\partial}{\partial \psi_{P}} I_{T}(\Psi) \\
& =2 \sum_{i}\left(\frac { 1 } { 2 } D _ { i } d _ { i } a _ { i + 1 } \operatorname { s i n } \alpha _ { i } \left\{-\frac{\psi_{i}-\psi_{0}}{d_{i}^{2}}+\left[\frac{\psi_{1+1}-\psi_{0}}{d_{i+1} \sin \alpha_{i}}-\frac{\psi_{i}-\psi_{0}}{d_{i} \tan \alpha_{i}}\right] \cdot\left[\frac{1}{d_{i+1} \sin \alpha_{i}}+\frac{1}{d_{i} \tan \alpha_{i}}\right]\right.\right. \\
& \left.\left.\quad+\sigma_{i}\left|a_{T_{i}}(P)\right| \psi_{0}\right)\right\}
\end{aligned}
$$




$$
\begin{aligned}
& =\sum_{i}\left\{D_{i} \frac{d_{1+1} \sin \alpha_{1}}{d_{1}}\left(\psi_{0}-\psi_{1}\right)+D_{1}\left(d_{i+1} \cos \alpha_{1}-d_{1}\right) \cdot\left[\frac{\psi_{1+1}-\psi_{0}}{d_{1+1} \sin \alpha_{1}}+\frac{\psi_{0}-\psi_{i}}{d_{1} \tan \alpha_{i}}\right]\right. \\
& \left.+2 \sigma_{i}\left|a_{T_{1}}(P)\right| \psi_{0}\right\} \\
& =\sum_{i}\left\{D_{1} \frac{a_{i+1}-d_{1} \cos \alpha_{i}}{d_{1} \sin \alpha_{1}}\left(\psi_{0}-\psi_{1}\right)+D_{i} \frac{d_{i}-d_{i+1} \cos \alpha_{i}}{d_{i+1} \sin \alpha_{1}}\left(\psi_{0}-\psi_{1+1}\right)\right. \\
& \left.+2 \sigma_{i}\left|a_{T_{1}}(P)\right| \Psi_{0}\right\}
\end{aligned}
$$

Comparing this with (2.6), we see that the lemma is proved.

It is not true in general that the matrix $A^{-1}$ is positive. Therefore, it is not true in general that the fundamental eigenvector in a multigroup problem has nonnegative components [9]. In order that $A^{-1}$ be positive, a sufficient condition is that the off-diagonal coefficients of $A$ be negative; i.e., for each mesh triangle $\mathrm{T}$,

$$
a_{i+1} \geq d_{i} \cos \alpha_{i}
$$

Since $d_{1} \cos \alpha_{1}$ is the length of the projection of the side $P P_{1}$ on the side $\mathrm{PP}_{1+1}$, a necessary and sufficient condition that (2.11) hold for each mesh triangle $T \in \mathcal{J}$, and for each angle $\alpha_{i}$ of $T$, is that each $T$ be an acute triangle. 


\section{The Coefficients of the Difference Equation}

It is desired to give formulas for the coefficients of the difference equation (2.6) in a form suitable for programming. For this, a mesh rectangle $T$ with vertices $P, Q$, and $R$ and associated quadrilaterals $a_{T}(P), a_{T}(Q)$, and $a_{T}(R)$ is depicted in Fig. 4。 It is desired to express all coefficients

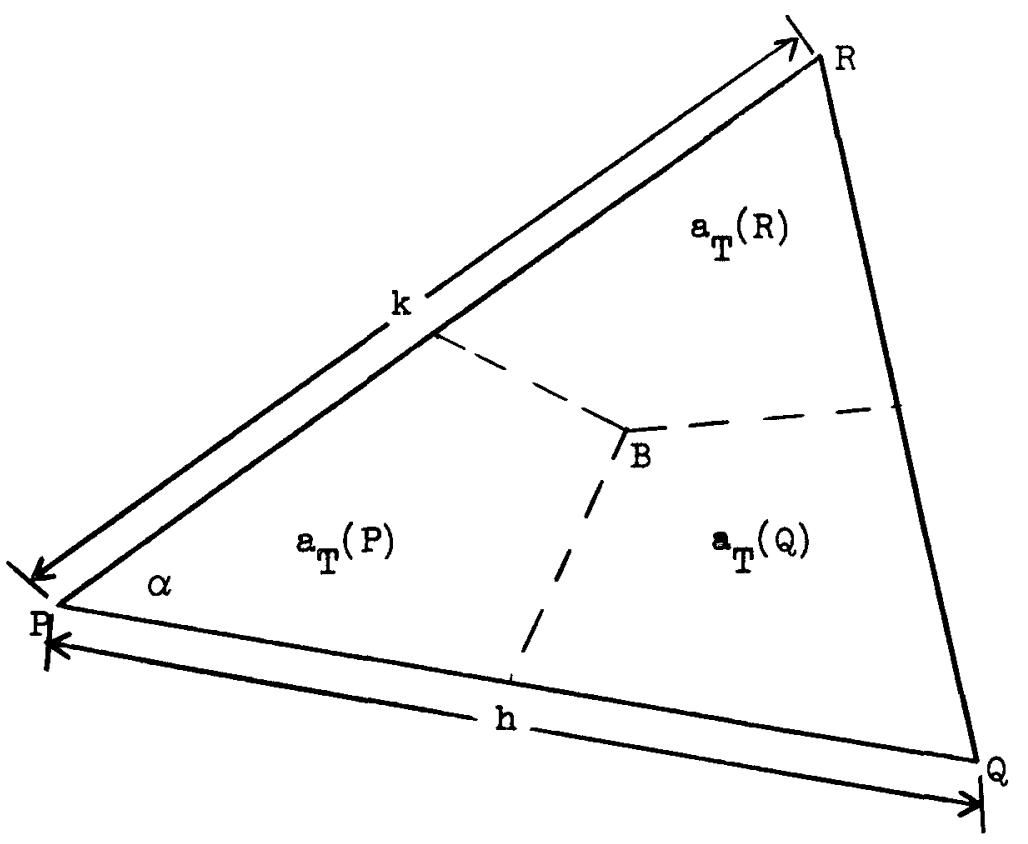

Figure 4

coming from mesh triangle $T$ in terms of the angle $\alpha$, the lengths $h$ and $k$, and the values $D_{T}$ and $\sigma_{T}$ of $D$ and $\sigma$ in $T$. The results are as follows.

$$
\begin{aligned}
& \phi_{P}-\phi_{Q} \text { coupling }=D_{T} \frac{k-h \cos \alpha}{2 h s i n \alpha} \\
& \phi_{P}-\phi_{R} \text { coupling }=D_{T} \frac{h-k \cos \alpha}{2 k s i n \alpha} \\
& \phi_{Q}-\phi_{R} \text { coupling }=\frac{1}{2} D_{T} \cot \alpha \\
& \phi_{P}-\phi_{P} \text { coupling }=\frac{1}{2} D_{T}\left[\left(\frac{h}{k}+\frac{k}{h}\right) \csc \alpha-2 \cot \alpha\right]+\sigma_{T}\left|a_{T}(P)\right|
\end{aligned}
$$




$$
\begin{aligned}
& \phi_{Q}-\phi_{Q} \text { coupling }=D_{T} \frac{k}{2 h} \csc \alpha+\sigma_{T}\left|a_{T}(Q)\right| \\
& \phi_{R}-\phi_{R} \text { coupling }=D_{T} \frac{h}{2 k} \csc \alpha+\sigma_{T}\left|a_{T}(R)\right| .
\end{aligned}
$$

In a multigroup calculation, the source $S$ comes from the solution of other neutron diffusion equations, and hence may be regarded as constant on each dodecagon $\hat{a}(P)$. In this case the right hand side of $(2.6)$ is $|\hat{a}(P)| S_{P}$.

To complete the speciflcation of the difference approximation, we indicate how to edit quantities of interest once the finite difference solution has been obtained. There are two types of edits, flux weighted cross section integrals, and leakage integrals. A flux weighted cross section integral is approximated by the formula

$$
\iint_{R_{1}} \tilde{\sigma} \phi d x d y=\sum_{T \in R_{1}} \tilde{\sigma}_{T}\left(\left|a_{T}(P)\right| \phi_{P}+\left|a_{T}(Q)\right| \phi_{Q}+\left|a_{T}(R)\right| \phi_{R}\right\}
$$

where the sum is taken over all mesh triangles $T \in \mathcal{J}$ which are contained In the edit region $R_{1}$, and where the mesh triangle $T$ is as depicted in Fig. 4. To edit the leakage out of a specified region $R_{1}$,

$$
-\oint_{\partial R_{1}} \underline{\mathrm{nn}} \cdot \nabla \phi \mathrm{ds}
$$

we will use (2.5) to approximate the line integral of $-\mathrm{Dn} \cdot \underline{\nabla} \phi$ along each side of the triangle $T$ of Fig. 4, where $\underline{n}$ is the outward pointing unit normal vector to the side. The formulas are 


$$
\begin{aligned}
& -\int_{P}^{Q} \underline{D} \cdot \nabla \phi d d s=\frac{\alpha}{k \sin \alpha}\left(\phi_{R}-\phi_{P}\right)+\cot \alpha\left(\phi_{Q}-\phi_{P}\right) \\
& -\int_{P}^{R} \underline{D} \cdot \nabla \phi d s=\frac{k}{h s i n \alpha}\left(\phi_{Q}-\phi_{P}\right)+\cot \alpha\left(\phi_{R}-\phi_{P}\right) \\
& -\int_{Q}^{R} \underline{D} \cdot \nabla \phi d s=\frac{k-h \cos \alpha}{h \sin \alpha}\left(\phi_{Q}-\phi_{P}\right)+\frac{h-k \cos \alpha}{k s i n \alpha}\left(\phi_{R}-\phi_{P}\right) .
\end{aligned}
$$

The difference equations used by PDQ-7 may now be specified by requiring for each mesh triangle $T$ that the point $B$ (see Fig. 4 ) be the centroid of the triangle. The resultant set of difference equations were also suggested by R. C. Gast. The centroid B may also be identified as the intersection of the 3 lines joining the vertices of $T$ to the mid points of the opposite sides. For this choice of B,

$$
\left|a_{T}(P)\right|=\left|a_{T}(Q)\right|=\left|a_{T}(R)\right|=\frac{1}{3}|T|=\frac{1}{6} \text { hksin } \alpha .
$$


IV. Derivation of the Difference Equations Using Discontinuous Trial Functions

In this section it is shown how to obtain the difference equations (2.6) for the boundary value problem (2.1),(2.2) by means of the selengutWachspress varlational principle $[10,11]$ which allows the use of discontinuous trial functions.

To define the variational princlple, we write (2.1) in the form

$$
\left\{\begin{array}{r}
\underline{J}+D \underline{\nabla} \phi=0 \\
\underline{\nabla} \cdot \underline{J}+\sigma \phi=s
\end{array}\right.
$$

Adapting the treatment of [10] to the inhomogeneous equation (4.1), we consider a set of functions $\phi, \phi^{t}, \underline{J}, \underline{J}^{*}$ defined on $R$, and we let $\mathcal{L}_{\text {int }}$ denote the totality of curves in $R$ on which $\phi$ or $\phi^{\text {th }}$ or both are discontinuous. $\mathscr{L}_{\text {int }}$ partitions $\mathrm{R}$ into a number of disjoint subregions $\mathscr{P}_{\mathrm{m}}$ in which $\phi$ and $\phi^{\text {th }}$ are continuous. At each point on $\mathscr{L}_{\text {int }}$ we choose unit normal $\underline{\text { n. Ietting }}$ + denote quantities on the side of $\mathcal{L}_{\text {int }}$ toward which $\underline{n}$ points, and letting

- denote evaluations on the other side, we consider the functional

$$
\begin{aligned}
& \tilde{F}\left(\phi, \phi^{*}, \underline{J}, \underline{J}^{*}\right)=\sum_{m} \int_{R_{m}} \int\left\{\phi \phi^{*}-D^{-1} \underline{J} \cdot \underline{J}^{*}-\underline{J}^{*} \cdot \nabla \phi-\underline{J} \cdot \nabla \phi^{*}\right. \\
& \left.-\phi s-\phi^{k} s^{\star}\right\} d x d y \\
& -\frac{1}{2} \oint_{\mathscr{L}_{\text {Int }}}\left\{\left(\underline{J}^{k}(+)+\underline{J}^{\star}(-)\right)(\phi(+)-\phi(-))+(\underline{J}(+)+\underline{J}(-)) \cdot\right. \\
& \left.\left(\phi^{\star}(+)-\phi^{\star}(-)\right)\right\} \cdot \underline{\text { nds }} \text {. }
\end{aligned}
$$


The quantities $\phi, J$ represent the approximate flux and current, and the quantities $\phi^{\#}, \underline{J}$ represent approximate adjoint flux and current.

If $\phi$ and $\underline{J}$ are such that $\mathcal{F}_{\text {is }}$ stationary under a variation of $\phi^{\text {th }}$ and $\underline{J}^{*}$, then on $\mathcal{L}_{\text {int }}$

$$
\begin{gathered}
\phi(+)=\phi(-) \\
\underline{\mathrm{n}} \cdot \underline{J}(+)=\underline{\mathrm{n}} \cdot \underline{J}(-)
\end{gathered}
$$

and $\phi$ and J satisfy $(4.1)$.

We now select a restricted family of trial functions for the flux and current, and apply stationarity of the reduced functional to get our difference approximation. A more general geometry will be used than in Section II in that the points $A_{1}$ of Fig. 2 will not necessarily lie on the mid points of the segments $\mathrm{PP}_{i}$.

For each mesh point $P$, we let $u_{P}$ be the function which is 1 in $\hat{a}(P) \cap R$ and $O$ elsewhere. Then

$$
\phi(x, y)=\sum_{P \in \ell_{0}} \phi_{P} u_{P}(x, y)
$$

$$
\phi^{*}(x, y)=\sum_{P=\&_{0}} \phi_{P}^{*} u_{P}(x, y)
$$

will be the trial functions for $\phi$ and $\phi^{\text {th }}$. For each mesh triangle $T$, let $v_{T}^{\prime}$ be the vector function which is 1 on $T$ and 0 elsewhere, and let $v_{T}^{\prime \prime}$ be the vector function which is $\underline{j}$ on $T$ and 0 elsewhere. Then 
$(4.4)$

$$
\begin{aligned}
& \underline{J}(x, y)=\sum_{T=\mathcal{T}}\left(J_{T}^{\prime} v_{T}^{\prime}(x, y)+J_{T}^{\prime \prime}\left(v_{T}^{\prime \prime}(x, y)\right),\right. \\
& \underline{J}^{t}(x, y)=\sum_{T=\mathcal{T}}\left(J_{T}^{\prime \prime} v_{T}^{\prime}(x, y)+J_{T}^{k \prime \prime} v_{T}^{\prime}(x, y)\right)
\end{aligned}
$$

will be the trial functions for $\underline{J}$ and $\underline{J}$.

With the finite fomilies of trial functions (4.3) and (4.4), 䖢 becomes

a function of the numbers $\phi_{P}, \phi_{P}^{k}, P \in \mathcal{L}_{O}$, and $J_{T}^{\prime}, J_{T}^{\prime \prime}, J_{T}^{*}, J_{T}^{\prime \prime}, T \in \mathcal{J}$. For a given $P$ (depicted in Fig. 2), F is linear in the variable $\phi_{P}^{*}$, and stationarity requires that

$$
\frac{\partial \sigma^{\sigma}}{\partial \phi_{P}^{k}}=\sum_{i}\left\{\int_{A_{i}}^{B_{i}} \underline{N}_{i}^{i} J d s+\int_{B_{i}}^{A_{i+1}} N_{i}^{1} J d s+\phi_{P} \sigma_{i}\left|a_{i}(P)\right|\right\}
$$

$$
-\iint_{\hat{\mathbf{a}}(P)} \operatorname{sdxdy}=0
$$

For a given $T$ (depicted in Fig. 5), F is linear in the variable $J_{T}^{* \prime}$, and stationarity requires that

$$
\frac{\partial \widetilde{\pi}}{\partial J_{T}^{A+1}}=-D_{T}^{-1}|T| J_{T}^{\prime}-\left(\phi_{Q}-\phi_{P}\right) \int_{A}^{D} \underline{i} \cdot N_{Q d} d s
$$

$$
-\left(\phi_{R}-\phi_{P}\right) \int_{B}^{D} \underline{i} \cdot \underline{N}_{b d} d s-\left(\phi_{R}-\phi_{Q}\right) \int_{C}^{D} \underline{i} \cdot \underline{N}_{c d} d s=0 .
$$

Finally, for a given $T, \mathcal{F}$ is linear in the variable $J_{T}^{\prime \prime}$, and stationarity requires that 
$(4.7)$

$$
\frac{\partial \mathcal{F}}{\partial J_{T}^{* \prime \prime}}=-D_{T}^{-1}|T| J_{T}^{\prime \prime}-\left(\phi_{Q}-\phi_{P}\right) \int_{A}^{D} \underline{j} \cdot N_{c d} d s
$$

$$
-\left(\phi_{R}-\phi_{P}\right) \int_{B}^{D} \underline{j} \cdot \underline{N}_{b d} d s-\left(\phi_{R}-\phi_{Q}\right) \int_{C}^{D} \underline{j} \cdot \underline{N}_{c d} d s=0 .
$$

Equations (4.5)-(4.7) comprise a set of difference equations which are given by the Selengut-Wachspress variational principle. Equation (4.5) is easily interpreted as the result of integrating the second equation of (4.1) around the dodecagon $\hat{a}(P)$. To interpret $(4.6)$ and $(4.7)$, we combine these two equations to obtain the vector equation

$$
\underline{J}=-\frac{D_{T}}{|T|}\left\{\left(\phi_{Q}-\phi_{P}\right) \overline{A D} \underline{N}_{a d}+\left(\phi_{R}-\phi_{P}\right) \overline{B D} \underline{N}_{b d}\right.
$$

$$
\left.+\left(\phi_{R}-\phi_{Q}\right) \overline{C D} \underline{N}_{c d}\right\}
$$

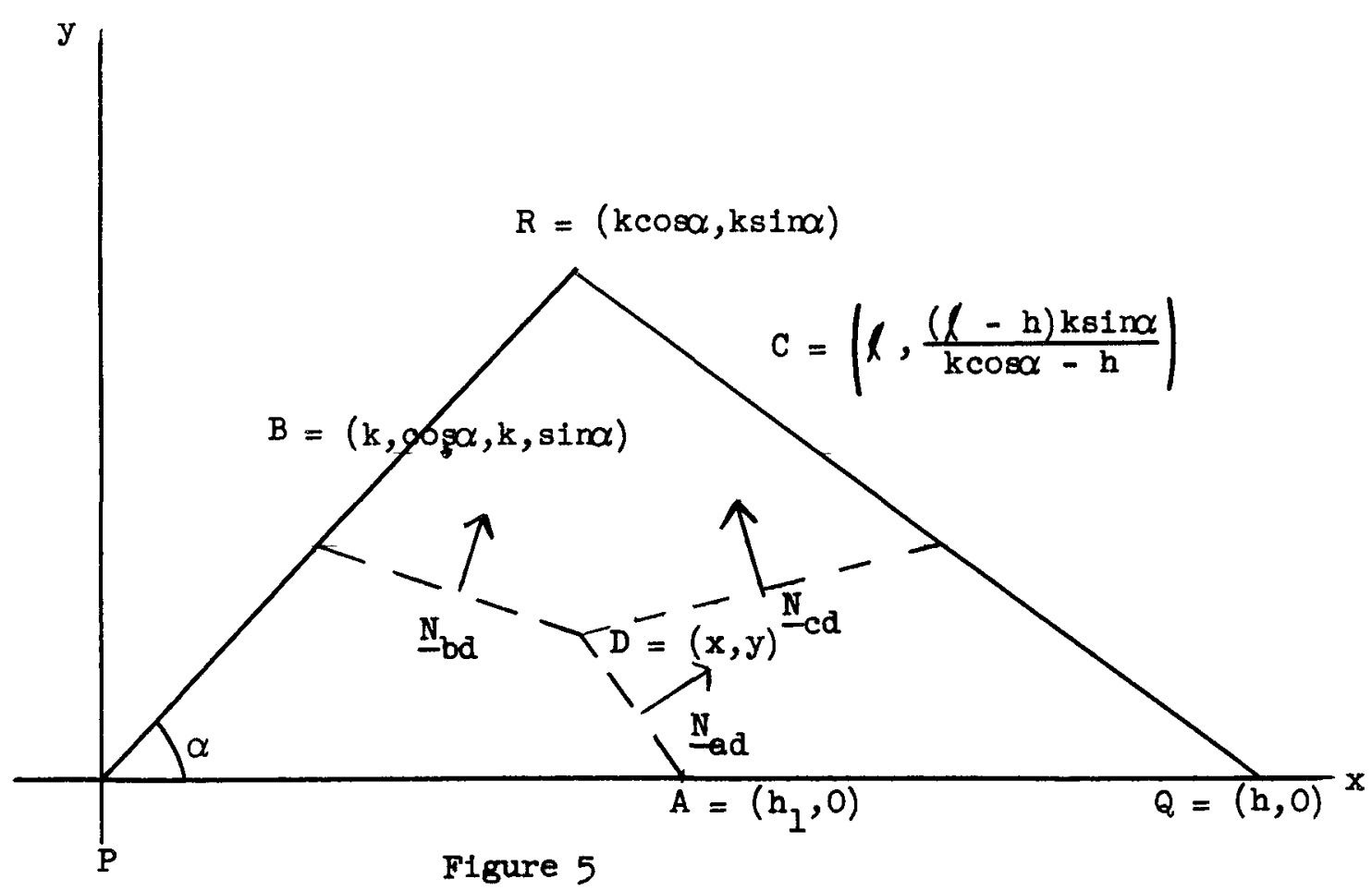


From Fig. 4 we see that $(4.8)$ is

$$
\begin{aligned}
& I=\frac{-D_{T}}{h k s i n \alpha}\left\{\left[\frac{(\ell-h) k \sin \alpha}{k \cos \alpha-h}\left(\phi_{Q}-\phi_{P}\right)+\left(k_{1} \sin \alpha-\frac{(\ell-b) k s i n \alpha}{k \cos \alpha-h}\right)\left(\phi_{R}-\phi_{P}\right)\right] \underline{1}\right. \\
& \left.+\left[\left(\mathrm{h}_{1}-\ell\right)\left(\phi_{\mathrm{Q}}-\phi_{\mathrm{P}}\right)+\left(\ell-\mathrm{k}_{1} \cos \alpha\right)\left(\phi_{\mathrm{R}}-\phi_{\mathrm{P}}\right)\right] \underline{\mathrm{j}}\right\} .
\end{aligned}
$$

In the case that $A, B$, and $C$ lie on the mid points of the sides of $T$, then

$$
\mathrm{h}_{1}=.5 \mathrm{~h}, \mathrm{k}_{1}=.5 \mathrm{k}, \ell=.5(\mathrm{~h}+\mathrm{k} \cos \alpha) \text {, }
$$

and we have

$$
\underline{J}=-D_{T}\left\{\frac{\phi_{Q}-\phi_{P}}{h} \underline{1}+\left[\frac{\phi_{R}-\phi_{P}}{k \sin \alpha}-\frac{\phi_{Q}-\phi_{P}}{\text { htan } \alpha}\right] \underline{j}\right\} .
$$

In this case, (4.11) agrees with the formula (2.5) for $\nabla \phi$ so that if $\mathrm{J}$ is put into (4.5), the difference equation (2.6) is obtained.

In the more general case, (4.9) gives a formula for $\nabla \phi$ which is not the same as (2.5). Therefore, in general the formula coming from (4.9) is not valid when $\phi(x, y)$ is a linear function of $x$ and $y$. It will turn out (Sec. V) that the truncation error of (2.6) is $O(1)$, that is, remains finite as the mesh spacings tend to 0 . With a general choice of $A, B$, and $C$, the truncation error $18 \mathrm{O}\left(\mathrm{b}^{-1}\right)$, that $1 \mathrm{~s}$, tends to infinity as the mesh spacings shrink to 0 . In this case, the Selengut-Wachspress variational principle gives a bad set of approximating equations. The general question is therefore of some importance: to find criteria on families of trial functions $\phi, \phi^{*}, \underline{J}, \underline{J}^{*}$, such that there is a unique pair of functions $(\phi, \underline{J})$ which are stationary points under a variation in F, and which tend to the solution of (4.1), (2.2) in some norm. 
The question might be raised what would happen if, with an arbitrary choice of points $A_{1}$, the integration technique were used to derive difference equations as in Section II. The answer is that the truncation error would be of the same order as when the $A_{1}$ are mid points but that the difference equations would no longer be symmetric. In other words, if the $A_{1}$ are placed at the mid points and then are caused to move off the mid point locations, the equations coming from the integration technique retain accuracy but lose symmetry, and the equations coming from the variational method retains symetry but lose accuracy.

It is appropriate to mention here another anonalous set of trial functions which has been obtained. The trial functions for $\phi$ and $\phi$ are those of (4.3). To describe the trial functions for $\underline{J}$ and $\underline{J}$, notice that if, in Fig. 2, the quadrilateral $Q_{i}$ is defined by the vertices $P B_{i} P_{i} B_{i-1}$, then these quadrilaterals do not overlap and fill space, and there is one such quadrllateral for each pir of neighboring mesh points $P Q$. The trial functions $\underline{J}(x, y)$ and $\underline{J}(x, y)$ are defined by defining them to be constant, but arbitrary, in each quadrilateral $Q$. The difference equations which results from the use of this set of trial functions in the Selengut-Wachspress varlational principle also has a truncation error which is $O\left(h^{-1}\right)$. 


\section{Truncation Error}

It will be shown that, in general the truncation error of the difference approximation $(2.6)$ is $O(1)$. To make this statement precise, let the mesh point $P$ be interior to a region of constant $D$ and $\sigma$. Then if both sides of $(2.6)$ are divided by $|\hat{a}(P)|$, we obtain

$$
\begin{aligned}
(I \phi)(P) \equiv & \frac{D}{\hat{a}(P)} \sum_{i=1}^{6}\left\{\frac{a_{i+1}-a_{i} \cos \alpha_{i}}{2 d_{i} \sin \alpha_{i}}\left(\phi_{0}-\phi_{i}\right)\right. \\
& \left.+\frac{a_{i}-a_{i+1} \cos \alpha_{1}}{2 d_{i+1} \sin \alpha_{i}}\left(\phi_{0}-\phi_{i+1}\right)\right\}+\sigma \phi_{0} \\
& =\frac{1}{\hat{a}(P)} \int_{\hat{a}(P)} \operatorname{saxdy} .
\end{aligned}
$$

$I \phi(P)$ should be an approximation to the differential operator $-D \nabla^{2} \phi+\sigma \phi$ at $P$. If

$$
L \phi=-D \nabla^{2} \phi+\sigma \phi \text { at } P
$$

whenever $\phi(x, y)$ is a polynomial of degree $r$ or less, but not when $\phi(x, y)$ is of degree $r+1$, then we say that the truncation error is $O\left(\mathrm{~b}^{\mathrm{r}-1}\right)$ at $P$ 。 Thus, we will show that $(5.2)$ holds whenever $\phi$ is linear function but not when $\phi$ is quadratic. (Of course, in special circumstances the truncation error may be smaller. For example the error is $O\left(h^{2}\right)$ at a mesh point $P$ if each $\mathrm{T}_{i}$ is an equilateral triangle and if each $\mathrm{B}_{1}$ is at the center of the triangle.) In spite of the truncation error being $O(1)$ it will be shown in the next section that the spproximate solution does converge to the true solution in a certain norm, as the mesh spacing tends to 0 . 
If $\phi$ is a linear function, then the approximation (2.5) is an equality, and (2.6) follows rigorously. Hence (5.2) is satisfied for linear functions.

To show that $(5.2)$ is not, in general, satisfied when $\phi$ is quadratic, let us take the case when the angle $\alpha_{1}=\angle \mathrm{PP}_{1} \mathrm{P}_{2}$ is $60^{\circ}$, the horizontal distance $\mathrm{PP}_{1}, \mathrm{P}_{4} \mathrm{P}_{1}$, etc. are all equal to $\mathrm{h}$, and the oblique distance $\mathrm{PP}_{3}, \mathrm{PP}_{6}$ etc. are all equal to $2 \mathrm{~h}$. Using the formulas for the coupling coefficients dereloped in section III, we get for (2.6) the equation

$$
\begin{aligned}
\sqrt{3}\left(\phi-\phi_{1}\right. & \left.+\frac{1}{3}\left(\phi-\phi_{2}\right)+\phi-\phi_{4}+\frac{1}{3}\left(\phi-\phi_{5}\right)\right)+2 h^{2} \sqrt{3} \sigma \phi \\
& =\iint_{\hat{\mathbf{a}}(P)} \operatorname{sdxdy}
\end{aligned}
$$

so that

$$
(L \phi)(P)=\frac{1}{2} h^{-2}\left\{\phi-\phi_{1}+\frac{1}{3}\left(\phi-\phi_{2}\right)+\phi-\phi_{4}+\frac{1}{3}\left(\phi-\phi_{5}\right)\right\} .
$$

If $P=(0,0), P_{1}=(n, 0)$, and if $\phi=x^{2}$, then

$$
\begin{aligned}
I \phi(P) & =\frac{D}{2} h^{-2}\left\{-2 h^{2}\right\}+\sigma \phi=-D, \\
-D \nabla^{2} \phi+\sigma \phi & =-2 D,
\end{aligned}
$$

so that $(5.2)$ is not satisfied. 
VI. Discretization Error

The purpose of this section is to show that the solution $\tilde{\phi}$ of the difference equations (2.7) tends in some sense to the solution $\phi(x, y)$ of the original boundary value problem $(2.1),(2.2)$ as the mesh spacing tends to 0 . This is especially important because of the result, contained in section $V$, that the truncation error does not necessarily tend to zero but may remain bounded as the mesh spacing tends to zero. The results of the present section are the only analytical means we have of showing that, despite a possibly large truncation error, the difference equations proposed here should give good results.

The proof given here is virtually the same as that of [3], in which a special case of our equations, the MacNeal equations, were discussed. Some of the notation of [3] will also be used. For example, if $E$ is a set of points in the $(x, y)$ plane and if $u$ is a real valued function defined on $\mathrm{E}$, we let

$$
\|u, E\|=\left[\iint_{E} u^{2} d x d y\right]^{1 / 2}
$$

denote the Hilbert space norm of $u$ over $E$. We also consider the norms

$$
\begin{aligned}
& \|u, E\|_{1}=\left[\|u, E\|^{2}+\left\|u_{x}, E\right\|^{2}+\left\|u_{y}, E\right\|^{2}\right]^{1 / 2}, \\
& \|u, E\|_{2}=\left[\|u, E\|_{1}^{2}+\left\|u_{x x}, E\right\|^{2}+\left\|u_{x y}, E\right\|^{2}+\left\|u_{y y}, E\right\|^{2}\right]^{1 / 2} .
\end{aligned}
$$

The area of $\mathrm{E}$ will be denoted by $|\mathrm{E}|$. For example $\mathrm{E}$ may in one instance be one of the mesh triangles, and in another situation $\mathrm{E}$ may be the entire region R. To use $\|\mathbf{u}, E\|_{1}$ and $\|u, E\|_{2}$ we must suppose that $u$ has a sufficient number 
of square integrable derivatives on $R$.

We will denote by $\bar{D}$ and $D$ the maximum and minimum values of the diffusion coefficient $D$, and by $\bar{\sigma}$ and $\underline{\sigma}$ the maximum and minimum values of the total cross section $\sigma$. It will be assumed that $\underline{\sigma}>0, \underline{D}>0$. The assumption on $\underline{g}$ is not really necessary, but simplifies the argument. We will let $\rho(P, Q)$ denote the distance between two points $P, Q$, and we let $c_{1}$ denote the diameter of the region $R$, defined by

$$
c_{1}=\sup \{\rho(P, Q), P, Q \in R\}
$$

Let $h(P)$ denote the maximum distance from a mesh point $P$ to any one of its neighboring mesh points. Let

$$
\bar{h}=\max _{P} h(P)
$$

be the maximum spacing. Oux first result proved in the same way as [3, Lemma 1].

Lemma 2. There is constant $c_{2}$ depending only on $c_{1}$ such that if $a_{T_{i}}(P)$ is the quadrilsteral shown in Fig. 3 , and if $u(x, y)$ has 2 derivatives on $a_{T_{1}}(P)$, then

$$
\left|\iint_{\mathbf{a}_{1}} u d x d y=u(P)\right| a_{\mathbb{T}_{i}}(P)|| \leq c_{2}\left|a_{T_{i}}(P)\right|\left\|u, a_{T_{i}}(P)\right\|_{2} .
$$

The next result is analogous to [3, Lemma 2], but the proof must be given separately. Let $T_{i}$ denote the triangle $P P_{i} P_{i+l}$ of Fig. 3. Let $L$ denote any one or the 3 line segments $A_{i} B_{i}, A_{i+1} B_{i}$, or $C B_{i}$, and let $N$ denote the corresponding unit normal vector as shown in Fig. 3. Finally, let $c_{3}>0$ be a positive number such that 


$$
c_{3}^{-1} \leq \frac{s}{t} \leq c_{3}
$$

where $s, t$ are the lengths of any 2 of the 3 sides of the triangle $T_{i}$ 。 (Thus $c_{3}$ is a bound on the "local ratio of mesh spacings".) Also suppose that

$$
c_{3} \geq|\csc \theta|
$$

where $\theta$ is the angle between any one of the line segments $A_{i} B_{i}, A_{i+1} B_{i}$, or $\mathrm{CB}_{1}$, and the corresponding side of the triangle, $\mathrm{PP}_{1}, \mathrm{PP}_{i+1}$, or $\mathrm{P}_{1} \mathrm{P}_{i+1}$ respectively. (This condition means that each of the line segments must stay away from lying on the corresponding side of the triangle. If $B_{1}$ is the center of gravity of the triangle, and if (6.2) holds, then $\mid$ cscel may be bounded by a function of $c_{3}$ itself.) It would be desirable to find error bounds that do not depend upon the quantity $c_{3}$.

Lemma 3. There is a positive constant $c_{4}$ depending only on $c_{3}$, such that if $u(x, y)$ has 2 derivatives on $T_{i}$, then (see $(2.4)$ )

$$
\left|\int_{L} \underline{\nabla} \cdot \underline{N} \mathrm{ds}-\int_{L} \underline{\nabla} \hat{u}_{\mathrm{T}_{i}} \cdot \underline{\mathrm{N}} \mathrm{ds}\right| \leq c_{4} \overline{\mathrm{h}}\left\|\mathrm{u}, \mathrm{T}_{i}\right\|_{2} .
$$

Proof. We will prove (6.4) when $L$ is the line segment $A_{i} B_{i}$; the same argument applies to the other two line segments. If $N_{i}^{\prime}$ is the $x$-component of the vector $\underline{N}_{i}$, we will show that there is a constant $c_{4}^{\prime}>0$ such that

$$
\left|\int_{A_{i} B_{i}} N_{i}^{\prime}\left(u_{x}-\frac{u_{i}-u_{0}}{d_{i}}\right) d s\right| \leq c_{4}^{\prime} \bar{h} \mid l u, T_{1} \|_{2} .
$$


An analogous argument would prove a similar inequality involving the $y$-component of $\underline{N}_{i}$, and this inequality, added to $(6.5)$ implies $(6.4)$. Thus, it suffices to prove $(6.5)$.

If $(x, y)$ is an arbitrary point of the triangle $T_{i}$, use of Taylor's theorem with the integral form of the remainder term gives

$$
\begin{aligned}
& u_{0}=u(x, y)-x u_{x}(x, y)-u y_{y}(x, y)+R_{0}(x, y) \\
& u_{i}=u(x, y)+\left(d_{i}-x\right) u_{x}(x, y)-y u_{y}(x, y)+R_{i}(x, y),
\end{aligned}
$$

where

$$
\begin{aligned}
R_{0}(x, y) & =\int_{t=0}^{1}(1-t)\left[x^{2} u_{x x}(\xi, \eta)+2 x y u_{x y}(\xi, \eta)+y^{2} u_{y y}(\xi, \eta)\right] d t, \\
\xi & =x(1-t), \eta=y(1-t),
\end{aligned}
$$

and

$$
\begin{gathered}
R_{i}(x, y)=\int_{t=0}^{1}(1-t)\left[\left(d_{i}-x\right)^{2} u_{x x}(\xi, \eta)-2 y\left(d_{i}-x\right) u_{x y}(\xi, \eta)\right. \\
\left.\quad+y^{2} u_{y y}(\xi, \eta)\right] d t, \\
\xi=d_{i} t+x(1-t), \eta=y(1-t) .
\end{gathered}
$$

Substituting the formules $(6.6),(6.7)$ for $u_{0}$ and $u_{i}$ into $(6.5)$, we find that (6.5) reduces to

$$
d_{i}^{-1}\left|\int_{A_{i} B_{i}}^{1} N_{i}^{\prime}\left[R_{i}-R_{0}\right] d s\right| \leq c_{4}^{\prime} \bar{h} \mid h u, T_{i} \|_{2} \text {. }
$$

The inequality $(6.8)$ will be broken into two parts. It will be shown that there is a positive constant $c_{4}^{\prime \prime}$ depending only on $c_{3}$ such that

$$
\mathrm{d}_{i}^{-1}\left|\int_{A_{1} B_{i}}^{1} N_{i}^{\prime} R_{0} d s\right| \leq c_{4}^{\prime \prime} \bar{h}|| u, T_{1} \|_{2} \text { 。 }
$$


An analogous inequality can be proved in the same way for the remainder term $R_{1}$, and these two inequalities can then be combined to imply (6.8). Thus the proof of the lemma is reduced to the proof of $(6.9)$. If the line $A_{i} B_{i}$ has the equation $x=\alpha y+\beta$, and if $B_{1}=\left(x_{1}, y_{1}\right)$, then $(6.8)$ becomes

$$
\begin{aligned}
& \frac{\sqrt{\alpha^{2}+1}}{d_{i}} \mid N_{1}^{\prime} \int_{y=0}^{y} \int_{t=0}^{1}(1-t)\left[(\alpha y+\beta)^{2} u_{x x}(\xi, \eta)\right. \\
& \left.+2 y(\alpha y+\beta) u_{x y}(\xi, \eta)+y^{2} u_{y y}(\xi, \eta)\right] d t d y\left|\leq c_{4}^{\prime \prime}\right| \mid u, T_{1} \|_{2} .
\end{aligned}
$$

We make a change of variables in the double integral from $(t, y)$ to $(\xi, \eta)$. It is seen that

$$
d \xi d \eta=-\beta(1-t) d t d y=-\frac{1}{2} d_{1}(1-t) d t d y
$$

since $A_{i}$ is at the mid point of the segment $\mathrm{PP}_{1}$. The range of integration in the $(\xi, \eta)$ plane is a subset of the triangle $T_{1}$. Also we may use the inequalities

$$
\begin{gathered}
|\alpha| \leq c_{3} \\
\left|N_{i}^{\prime}\right| \leq 1 \\
|\beta|,|y| \leq \bar{h} \\
\frac{|y|}{d_{i}}, \frac{\beta}{d_{1}} \leq c_{3} .
\end{gathered}
$$

We find that the left hand side of $(6.10)$ is bounded by the quantity 


$$
c_{4}^{\prime \prime \prime} \int_{T_{i}} \int\left[\left|u_{x x}\right|+\left|u_{x y}\right|+\left|u_{y y}\right|\right] d \xi d \eta
$$

where $c_{4}^{\prime \prime \prime}$ depends only on $c_{3}$. Applying Schwartz's inequality to the integral in (6.11), we learn that the left hand side of (6.10) is bounded by

$$
\begin{aligned}
& c_{4}^{\prime \prime \prime} \sqrt{3} \iint_{T_{i}} 1 \cdot\left[u_{x x}^{2}+u_{x y}^{2}+u_{y y}^{2}\right]^{1 / 2} d_{\xi} d \eta \\
& \leq c_{4}^{\prime \prime \prime} \sqrt{3}\left|T_{i}\right|^{1 / 2} \cdot\left\|u, T_{i}\right\|_{2} \\
& \leq c_{4}^{\prime \prime \prime} \sqrt{1.5} \bar{n}\left\|u, T_{i}\right\|_{2} .
\end{aligned}
$$

This proves (6.10) and completes the proof of Lemma 3 .

We now turn our attention to the set of vectors $C_{O}$, defined in section II. We introduce two inner products on $C_{0}$. If $\alpha, \beta \in C_{0}$ these are defined by

$$
\begin{aligned}
& (\alpha, \beta)=\sum \hat{l} \hat{Q}(P) \cap R \mid \alpha_{P} \beta_{P} \\
& (\alpha, \beta)_{I}=(\alpha, \beta)+\sum_{I}\left(\alpha_{P}-\alpha_{Q}\right)\left(\beta_{P}-\beta_{Q}\right) .
\end{aligned}
$$

The sum $\sum$ is taken over all mesh points $P \in \mathscr{S}_{O}$, and the sum $\Sigma_{1}$ is taken over all pairs of neighboring mesh points $P, Q \in \&$. The norms corresponding to these inner products are denoted by $\alpha$ and $\|\alpha\|_{1}$ respectively. These norms are finite difference analogues of the norms $\|u, R\|$ and $\|u, R\|_{1}$ introduced above.

These inner products should not be confused with the ordinary inner product on $C_{O}$, defined by considering $\underline{\alpha} \in C_{O}$ as a column vector and writing 


$$
\underline{\alpha}^{T} \underline{\alpha}=\sum \alpha_{P}^{2}
$$

A relation between the various inner products is given by the following lemma .

Lemma 4. There is a positive constant $c_{5}$ depending only on $c_{3}$ and the upper and lower bounds for $D$ and $\sigma$ such that if $\underline{\Psi} \in C_{0}$,

$$
c_{5}^{-1}\|\Psi\|_{1}^{2} \leq \Psi^{\mathrm{T}} \mathrm{A}_{\underline{4}} \leq c_{5}\|\Psi\|_{1}^{2} .
$$

Proof. Let $\mathrm{T}$ be mesh triangle, and let the coordinate system be chosen so that $T$ is situated as in Fig. 3 , with $\alpha_{i}$ an angle of satisfying

$$
\alpha_{i} \geq 60^{\circ}
$$

Then using (2.9),

$$
\begin{aligned}
I_{T}(\Psi) & \leq \frac{1}{2} \bar{D} a_{i} d_{i+1}\left\{d_{i}^{-2}\left(\psi_{i}-\psi_{0}\right)^{2}+\frac{2}{d_{i+1}^{2} \sin ^{2} \alpha_{i}}\left(\psi_{i+1}-\psi_{0}\right)^{2}\right. \\
& \left.+\frac{2}{d_{i}^{2} \tan ^{2} \alpha_{i}}\left(\psi_{1}-\psi_{0}\right)^{2}\right\} \\
& +\bar{\sigma}\left\{\left|a_{T_{i}}(P)\right| \psi_{0}^{2}+\left|a_{T_{i}}\left(P_{i}\right)\right| \psi_{i}^{2}+\left|a_{T_{i}}\left(P_{i+1}\right)\right| \psi_{i+1}^{2}\right\} \\
& \leq \frac{1}{2} \bar{D}_{3}^{2}\left[\left(\psi_{i}-\psi_{0}\right)^{2}+\frac{8}{3}\left(\psi_{i+1}-\psi_{0}\right)^{2}+\frac{4}{3}\left(\psi_{i}-\psi_{0}\right)^{2}\right] \\
& +\bar{\sigma}\left(\left|a_{T_{i}}(P)\right| \psi_{0}^{2}+\left|a_{T_{i}}\left(P_{i}\right)\right| \psi_{i}^{2}+\left|a_{T_{i}}\left(P_{i+1}\right)\right| \psi_{i+1}^{2}\right\} .
\end{aligned}
$$


Using (2.10), we find that the right hand inequality of (6.14) has been proved with

$$
c_{5} \geq \max \left\{\frac{7}{3} \bar{D} c_{3}^{2}, \bar{\sigma}\right\}
$$

To prove the other inequality we note that

$$
\|\Psi\|_{I} \leq \sum_{T \in J}^{\prime} \bar{I}_{T}(\Psi)
$$

where the quadratic form $\bar{I}_{T}(\Psi)$ is defined, for each mesh triangle $T \in J$, by

$$
\begin{aligned}
\bar{I}_{T}(\psi) & =\left(\psi_{0}-\psi_{1}\right)^{2}+\left(\psi_{0}-\psi_{i+1}\right)^{2}+\left(\psi_{i}-\psi_{i+1}\right)^{2} \\
& +\left|a_{T_{i}}(P)\right| \psi_{0}^{2}+\left|a_{T_{i}}\left(P_{i}\right)\right| \psi_{i}^{2}+\left|a_{T_{i}}\left(P_{i+1}\right)\right| \psi_{i+1}^{2} .
\end{aligned}
$$

We will show that there is a positive quantity $c_{5}^{\prime}$ depending only on $c_{3}$ such that for each $T \in \sigma$,

$$
\bar{I}_{T}(\Psi) \leq c_{5}^{\prime} I_{T}(\Psi)
$$

The inequality $(6.17)$, when summed over $T \in J$ and coupled with $(6.16)$ and $(2.10)$, implied the left hand inequality of (6.14) provided that

$$
c_{5} \geq c_{5}^{\prime}
$$

Hence, to prove the lemma it suffices to prove (6.17). We have, using Schwartz's inequality 


$$
\begin{aligned}
\left(\psi_{1}-\psi_{0}\right)^{2} & +\left(\psi_{1+1}-\psi_{0}\right)^{2}+\left(\psi_{i+1}-\psi_{1}\right)^{2} \\
& =\left(\psi_{1}-\psi_{0}\right)^{2}+\left(\psi_{1+1}-\psi_{0}\right)^{2}+\left(\psi_{1+1}-\psi_{0}+\psi_{0}-\psi_{1}\right)^{2} \\
& \leq " 3\left(\psi_{1}-\psi_{0}\right)^{2}+3\left(\psi_{1+1}-\psi_{0}\right)^{2} .
\end{aligned}
$$

Also,

$$
\begin{aligned}
\psi_{1+1}-\psi_{0} & =d_{i+1} \sin \alpha_{i}\left(\frac{\psi_{i+1}-\psi_{0}}{d_{i+1} \sin \alpha_{i}}-\frac{\psi_{1}-\psi_{0}}{d_{i} \tan \alpha_{i}}\right) \\
& +d_{i+1} \cos \alpha_{i} \cdot \frac{\psi_{i}-\psi_{0}}{d_{i}}
\end{aligned}
$$

so

(6.19) $\left(\psi_{1+1}-\psi_{0}\right)^{2} \leq d_{1+1}^{2}\left[\left(\frac{\psi_{1+1}-\psi_{0}}{d_{1+1} \sin \alpha_{1}}-\frac{\psi_{1}-\psi_{0}}{d_{1} \tan \alpha_{1}}\right)^{2}+\frac{1}{d_{i}^{2}}\left(\psi_{1}-\psi_{0}\right)^{2}\right]$.

Combining (6.18) and (6.19), we find that

$$
\begin{aligned}
\left(\psi_{1}-\psi_{0}\right)^{2} & +\left(\psi_{1+1}-\psi_{0}\right)^{2}+\left(\psi_{1+1}-\psi_{1}\right)^{2} \\
& \leq 3\left(d_{1}^{2}+d_{1+1}^{2}\right)\left[\frac{1}{d_{1}^{2}}\left(\psi_{1}-\psi_{0}\right)^{2}+\left(\frac{\psi_{1+1}-\psi_{0}}{d_{1+1} \sin \alpha_{1}}-\frac{\psi_{1}-\psi_{0}}{d_{1} \tan \alpha_{1}}\right)^{2}\right] .
\end{aligned}
$$

Using (6.2) and (6.15)

$\cdot(6.20)$

$$
\begin{aligned}
& \frac{3\left(d_{i}^{2}+d_{i+1}^{2}\right)}{D_{1} \mid T_{i}} \leq \underline{D}^{-1} \frac{3\left(d_{1}^{2}+d_{i+1}^{2}\right)}{\frac{1}{2} d_{i} d_{i+1} \cdot \frac{1}{2} \sqrt{3}} \\
& \leq 8 \sqrt{3} c_{3} D^{-1} .
\end{aligned}
$$

30 
Inequalities $(6.19)$ and $(6.20)$ combine to prove $(6.17)$ with

$$
c_{5}^{\prime}=8 \sqrt{3} c_{3} \underline{D}^{-1}
$$

This completes the proof of Lemma 4.

We can now give the estimate of the discretization error. For this we let $\phi(x, y)$ be the solution of the boundary value problem, and we let $\phi \in C_{0}$ be the vector defined by $\phi_{P}=\phi(P), P \in \mathscr{f}_{0^{\circ}}$ If $\tilde{\phi}$ is the solution to the difference equations $(2.7)$, we define the error vector $e \in C_{0}$ by $\underline{e}=\underline{\underline{\varepsilon}}-\tilde{\phi}$.

Theorem. Suppose that the solution $\phi(x, y)$ of $(2.1),(2.2)$ has continuous partial derivatives of the second order in each mesh triangle $T$. Then there is a positive constant $c_{6}$ depending only on $c_{2}, c_{3}$ and the upper and lower bounds for $\sigma$ and $D$, such that

$$
\|\underline{e}\|_{1} \leq \bar{h}_{6}\|\phi, R\|_{2}
$$

Proof. We will show that there is a positive constant $c_{6}^{\prime}$ depending on the same quantities as $c_{6}$, such that

$$
\underline{e}^{\mathrm{T}} \underline{\mathrm{Ae}} \leq \overline{\mathrm{h}} \mathrm{c}_{6}^{\prime}\|\underline{e}\|_{1}\|\phi, \mathrm{R}\|_{2}
$$

When (6.22) is established, use of (6.14) with $\Psi$ replaced by $e$ will show that (6.21) holds with $c_{6}=c_{5} c_{6}^{\prime}$.

since $\underline{e}=\underline{\phi}-\underline{\phi}$, at any mesh point $\mathrm{P} \in \mathcal{\&}_{0}$,

$$
\begin{aligned}
\left(\underline{A e}_{\mathrm{P}}\right. & =(\mathrm{A} \underline{\phi})_{\mathrm{P}}-\left(\tilde{A}_{\underline{\phi}}\right)_{\mathrm{P}} \\
& =(\mathrm{A} \underline{\phi})_{\mathrm{P}}-\underline{\mathrm{S}}_{1, \mathrm{P}} .
\end{aligned}
$$


Now if $P$ is an interior mesh point, the usual technique of integrating by parts gives

$$
\begin{aligned}
& S_{1, P}=\iint_{\hat{Q}(P)} s(x, y) d x d y
\end{aligned}
$$

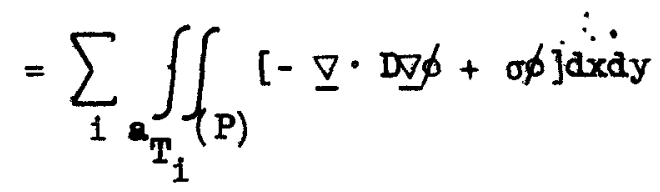

$$
\begin{aligned}
& =\sum_{i}\left[D_{1} \int_{A_{1} B_{i}} \underline{N}_{i} \cdot \underline{\nabla}_{1} \phi d \mathrm{~d} s+\int_{B_{i} A_{1+1}} \underline{N}_{i+1} \cdot \nabla \phi d s\right] \\
& +\sum_{i} \sigma_{i} \iint_{a_{T_{i}}} \phi d x d y
\end{aligned}
$$

Comparing this with (2.6) and defining the error terms

$$
\begin{aligned}
& E_{i}^{\prime}(P)=D_{i} \int_{A_{i} B_{i}} \underline{N}_{i}^{\prime}\left(\nabla \hat{\phi}_{T_{i}}-\nabla \phi\right) d s, \\
& E_{i}^{\prime \prime}(P)=D_{i} \int_{B_{1} A_{i+1}} \underline{N}_{i}^{\prime \prime}\left(\nabla \hat{\phi}_{T_{1}}-\nabla \phi\right) d s, \\
& F_{i}(P)=\sigma_{i}\left[\left|a_{T_{i}}(P)\right| \phi_{P}-\int_{a_{T_{i}}} \phi d x d y\right],
\end{aligned}
$$

it is seen that

$$
(A C)_{P}=\sum_{i}\left[E_{i}^{\prime}(P)+E_{i}^{\prime \prime}(P)+F_{i}(P)\right] \text {. }
$$


The same definitions will hold when $P$ is a boundary point except that those indices $i$ for which $T_{i}$ lies outside $R$ will not be considered. If (6.23) is multiplied by $e(P)$ and summed over all $P \in \mathscr{S}_{O}$, it is seen that each error $E_{i}(P)$ occurs as a factor in two terms, once in the term $e_{p} E_{i}^{\prime}(P)$, and the other time in the term $-e_{P_{i}} E_{i}^{\prime}(P)$. Hence

$$
\sum e_{P} E_{i}^{\prime}(P)=\sum_{l}\left(e_{P}-e_{P_{i}}\right) E_{i}^{\prime}(P)
$$

where the sum $\Sigma$ is taken over all mesh points $P \in \mathcal{S}_{0}$, and where the sum $\Sigma_{1}$ is taken over all pairs of mesh points $P, P_{i} \in \mathcal{S}$ such that $P$ and $P_{1}$ are neighbors and such that, if the neighboring points $P$ and $P_{i}$ are placed as in Fig. 2, the mesh triangle $T_{i}$ lies inside $\bar{R}$. A similar argument shows that

$$
\sum e_{P} E_{i}^{\prime \prime}(P)=\sum_{l}\left(e_{P}-e_{P}\right) E_{i+1}^{\prime \prime}(P)
$$

Using (6.24) and (6.25) we find that

$$
\underline{e}^{\mathrm{T}} \underline{\underline{e}}=I+I I+I I I
$$

where the quantities I, II, and III are defined by

$$
\begin{aligned}
I & =\sum_{1}\left(e_{P}-e_{P_{i}}\right) E_{i}^{\prime}(P) \\
I I & =\sum_{1}\left(e_{P}-e_{P_{i+1}}\right) E_{i}^{\prime \prime}(P), \\
I I I & =\sum_{i, P} e_{P} F_{i}(P) .
\end{aligned}
$$


To prove (6.22) it will be shown that each of the 3 quantities $|I|,|I| \mid$, and |III| satisfies a bound similar to (6.22). To bound I, we use Lemma 3 and Schwartz's inequality to obtain

$$
\begin{aligned}
|I| & \leq c_{4} \overline{\mathrm{hD}} \Sigma_{1}\left|e_{P}-e_{P_{1}}\right|\left\|\phi, T_{1}\right\|_{2} \\
& \leq c_{4} \overline{\mathrm{Dh}}\left\{\Sigma_{1}\left(e_{P}-e_{P_{1}}\right)^{2}\right\}^{1 / 2}\left\{\sum_{T \in \mathcal{J}}\|\phi, T\|_{2}^{2}\right\}^{1 / 2} \\
& \leq c_{4} \overline{\mathrm{Dh}} \mid \mathrm{le}\left\|_{1}\right\| \phi, \mathrm{R} \|_{2} .
\end{aligned}
$$

A similar argument shows that

$$
|I I| \leq c_{4} \overline{D h}\|e\|_{1}\|\phi, R\|_{2}
$$

Finaliy, use of Lema 2 and Schwartz's inequality shows that

$$
\begin{aligned}
& I I I \leq \overline{\sigma c} \sum_{P, 1}\left|a_{T_{1}}(P)\right|\left|e_{P}\right| \cdot\left\|\phi, a_{T_{1}}(P)\right\|_{2} \\
& \leq \bar{\sigma} c_{2} \bar{h} \sum_{P, i}\left|a_{T_{i}}(P)\right|^{1 / 2}\left|e_{p}\right| \cdot\left\|\phi, a_{T_{1}}(P)\right\|_{2} \\
& \leq \bar{\sigma} c_{2} \bar{h}\left\{\sum_{P, 1} a_{T_{1}}(P) e_{P}^{2}\right\}^{1 / 2}\left\{\sum_{P, 1}\left\|\phi, a_{T_{1}}(P)\right\|_{2}^{2}\right\}^{1 / 2} \\
& \leq \overline{\sigma c} c_{2} \text { hlle }\|\| \phi, R \|_{2} \\
& \leq \overline{a c_{2}} \bar{h}||\left\|_{1} \mid \phi \phi, R\right\|_{2} \text {. }
\end{aligned}
$$

Use of these bounds for I, II, and III proves (6.22) with

$$
c_{6}^{\prime}=2 c_{4} \bar{D}+\bar{\sigma} c_{2}
$$

and completes the proof of the theorem.

34 
Several comments must be made concerning the hypothesis on the existence of derivatives of $\phi(x, y)$ that is made in the theorem. It would be desirable to make no such assumption concerning the derivatives but merely to assert that any solution satisfies the hypothesis and hence satisfies the error estimate (6.21). If true, this would be a fact concerning the solution of multiregion problems. Although this is plausible, we do not know how to prove it.

In the proof of the theorem it is really not necessary to assume that $\phi$ has continuous second derivatives in each mesh triangle. One may weaken this requirement somewhat by assuming only that $\phi$ is a limit of a sequence of functions $\phi_{n}$ having continuous second derivatives in each mesh triangle, and that each derivative of $\phi$ of order $\leq 2$ is a limit in the square integrable sense of the sequence of corresponding derivatives of the $\phi_{n}$. This weaker statement is more plausible but we still do not know how to prove it.

If $D$ and $\sigma$ are constant on $R$ (that is, if we have a one region problem) if $R$ is convex, and if the boundary condition (2.2) then it may be shown that the weaker hypothesis on $\phi$ is valid. In fact one may prove

$$
\|\phi, R\|_{2} \leq \mathrm{cls}, \mathrm{R} \|
$$

With this inequality the error bound (6.21) becomes an a priori error bound. In this case one can imagine a technique for determining the optimal location of the auxiliary points $B_{1}$, as follows. The error vector $\underline{e}$ is obviously a linear function of $S$, since $\oint$ and $\Psi$ are linear functions of $S$. Because of (6.21) and (6.27), the error $e_{P}$ at each mesh point is a bounded linear functional of $S$. Letting $S$ range over the Hilbert space of square integrable 
functions on $\mathrm{R}$, by the Riesz representation theorem there is a function $K_{P}(x, y)$ such that

$$
e_{P}=\iint_{R} S(x, y) K_{P}(x, y) d x d y
$$

In particular,

$$
\sup \frac{\|\underline{\underline{e}}\|}{\|S, R\|}=\left\{\iint_{R} K(x, y)^{2} d x d y\right\}^{l / 2}
$$

where

$$
\mathbf{K}(\mathrm{x}, \mathrm{y})=\left\{\sum_{\mathrm{P}} \mathrm{K}_{\mathrm{P}}(\mathrm{x}, \mathrm{y})^{2}\right\}^{1 / 2}
$$

The kernel $\mathrm{K}(\mathrm{x}, \mathrm{y})$ also depends on the points $\mathrm{B}_{1}$. Therefore the norm

$$
\left\{\iint_{R} K(x, y)^{2} d x d y\right\}^{1 / 2}
$$

is a function of a finite number of parameters, representing the coordinates of the $B_{i}$. If this norm is minimized with respect to these parameters a choice of points $B_{i}$ would be found whtch is optimal in some sense. 


\section{REFERENCES}

1. Allan M. Winslow, "Numerical Calculation of Static Magnetic Fields in an Irregular Triangle Mesh, ${ }^{8}$ UCRL-7748, August 1964, Lawrence Radiation Laboratory, Livermore, California.

2. R. H. MacNeal, "An Asymetrical Finite Difference Network," Quart. Appl. Math。, v。 12 (1953), pp。295-310.

3. R. B. Kellogg, "Difference Equations on a Mesh Arising From a General Triangulation," Math. of Comp. Vol. 18 (1964), pp. 203-210.

4. Wo R。 Cadwell, Po F。 Buerger, and C.J. Pfeifer, "The PDQ-5 and PDQ-6 Programs for the Solution of the Two-Dimensional Neutron Diffusion-

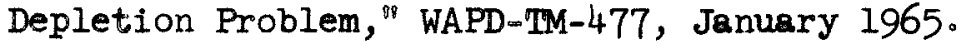

5. J. L. Synge, "The Hypercircle in Mathematical Physics," Cambridge University Press, Cambridge, England (1957)。

6. R。 B. Kellogg, "A Ritz Finite Difference Approximation to the Neutron Diffusion Equation," WAPD-BT-31, 1964.

7. R. B. Kellogg, "Ritz Difference Equations on a Triangulation," ANL-7050, (Proceedings of the Conference on the Application of Computing Methods to Reactor Problems.) August 1965.

8. A. A. Gonzalez and B. H. Ruth, "A Method for Numerical Solution of the Neutron-Diffusion Equations in Tridirectional (hexagonal) Geometry with a Variable Spatial Mesh," Trans. Am. Nucl. Soc. Vol 6 (1963), pp. 282-283.

9. G. Birkhoff and R。S. Varga (1958), "Reactor Criticality and Non-

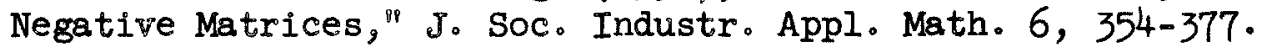

10. J. B. Yasinsky and S. Kaplan, "Synthesis of Three-Dimensional Flux Shapes Using Discontinuous Sets of Trial Functions," WAPD-T-1874 (to be published in Nuc. Sci. and Eng.)

11. E。 L。 Wachspress and M。 Becker, "Variational Synthesis with Discontinuous Trial Functions, ${ }^{8}$ ANL-7050, (Proceedings of the Conference on the Applicstion of Computing Methods to Reactor Problems.) August 1965. 